\title{
En torno al sueño infinito de Patricio Núñez Henríquez (1938 - 2017)
}

\author{
Lautaro Núñez ${ }^{1}$
}

Cuando las piedras oyen mi paso

Sienten una ternura que les ensancha el alma

Se hacen señas furtivas y hablan bajo:

Allí se acerca el buen amigo

El hombre de las distancias

Que viene fatigado de tanta muerte al hombro

De tanta vida en el pecho

Y busca donde pasar la noche (Huidobro 1948).

\section{Los Inicios}

Nació el 10 de enero de 1938 y por lo mismo se asumía más viejo por 14 días del suscrito, pero de inmediato admitía que era de los "Núñez del Sur". Habitante de la educación estatal, amigo de los deportes fuertes como el rugby, joven viajero precursor de los "mochileros", en tanto que ya tempranamente conocía los países vecinos. Su primera etapa en términos de "formación de cuadros" ocurrió en su propia casa familiar, donde leyó todo aquello progresista que su padre y hermanos le dejaban a mano. Paralelamente se leyó todos los textos de historia y no dejó mapa que no dibujara, confidenciándome un día que tuvo la audacia, estando en el liceo, de aspirar a escribir una historia de Chile.

Su padre en su tiempo libre era periodista corresponsal de Chile para los países socialistas. A su madre una vez la ví sola con un letrero entre una multitud en el Parque Forestal, reclamando por alguna causa justa. Sus hermanos, todos marcados por la "zurda" y desde jóvenes "ciudadanos del mundo" se dispersaron formados bajo una notable y genuina cultura progresista: Guillermo, Premio Nacional de Artes Plásticas, académico exiliado y activo militante anti dictadura. Sergio se fue al exilio durante la presidencia de Carlos Ibáñez y no volvió. Fundó un taller de arte social aplicado en Praga con sus afiches de gloria y fama, cuya hija checa es sobrina de Patricio. Carlos, hombre de teatro y la mano derecha del Premio Nacional de Arte, Pedro de la Barra, trasladado con su equipo a la Universidad de Chile de Antofagasta para replicar su modelo "experimental". Jorge en el arte radial, al tanto que María Angélica y Patricia iban por las mismas sendas de la creación y percepción del progreso social. Con Inger Kock criaron a sus hijos Rodrigo, médico veterinario, y Carolina unida al arte y la estética.

\section{Su Formación Académica}

Su segunda marca sociopolítica ocurrió en la mejor escuela de formación de cuadros: el Instituto Pedagógico de la Universidad de Chile durante los tiempos de absoluta gratuidad y libertad de pensamiento, con un elenco de profesores inolvidables que nos enseñaron en ciencia y conciencia cómo pensar y transformar el mundo: Hernán Ramírez, Fernando Ortíz (desaparecido), Bernardo Berdichewsky, César de León, Rafael Barahona, Carlos Munizaga, Juan Munizaga (Centro de Estudios Antropológicos) y Olga Poblete, Presidenta del Consejo Chileno de la $\mathrm{Paz}$, entre otros de igual renombre. Frente a tanta inteligencia, en su mayoría progresista, se explica por qué la dictadura le quitara el Pedagógico a la Universidad de Chile, donde se multiplicaba este laboratorio ideológico con generaciones que aspiraban a cambios francamente radicales.

Patricio vivió allí cuando las juventudes solían desprenderse de los partidos tradicionales de izquierda para abordar cuestiones de esos tiempos que también se reflejaban en las marchas y los sucesos que repercutían en el mundo. Todo conducía a visibilizar los tiempos de cambios: El Movimiento del 26 de Julio (1953) que levantaba la revolución cubana, las protestas de las universidades europeas conducentes a la Revolución de Mayo de Paris que rebotaban en Chile (1968). El movimiento de la Primavera de Praga (1968) se acallaba con la ocupación soviética. Surgían los istmos, cada uno con sus diarios murales en los pasillos del Pedagógico, y las ansias de ganar las elecciones del Centro de Alumnos. Patricio creía más en los partidos de izquierda tradicionales, puesto que venía de una educación política familiar excepcional, donde el comunismo y sus principios marxistas eran un tema de lectura y de comentario diario. Sin embargo, todos juntos, comunistas,

\footnotetext{
${ }^{1}$ Instituto de Arqueología, Antropología y Museo, Universidad Católica del Norte, San Pedro de Atacama, Chile. lautaro.nunez@hotmail.com
} 
socialistas, radicales, MIR, MAPU, FER y otros movimientos emergentes salíamos a bajarle los suspensores a los trolebuses para armar los tacos en Irarrázaval que respondían a la protesta de turno.

Ciertamente, Patricio vivió en la capital, entre los años 1960-1973, las aspiraciones de cambios cuando los universitarios gestaron un movimiento estudiantil que reflejaba el descontento mundial: "Seamos realistas, hagamos lo imposible", creciendo de esta manera una actitud contestataria con enorme disciplina doctrinaria entre festivales y algo de la simbología hippie con ropas ad hoc y amores más desatados entre ofrendas de flores. No estoy muy seguro, si de este contexto viene esa imagen invariable de Patricio con short, zapatillas "condorito", a "cuero pelado", pelo largo y copiosa barba que mantuvo siempre hasta el final junto a su riguroso materialismo histórico.

Los tiempos previos al año 1973 fueron en verdad muy estimulantes para los universitarios. En el año 1967 se había gestado la reforma universitaria para democratizar las universidades. Se exigían más derechos sociales, no más sangre en Vietnam y la guerrilla del Che Guevara se trasladaba a Bolivia. Más cerca se activaban las campañas pro Allende y se escuchaban innovadoras conferencias sobre materialismo histórico y la arqueología social se afirman en Concepción y Santiago con la presencia de Luis Lumbreras y Julio Montané. Todos pensaban reformar aún más a la Universidad de Chile, pero sin dejar de reflexionar sobre cómo activar los movimientos de cambios para el futuro del país y de Latinoamérica. Entre esas marchas nunca olvidaremos ese letrero de un MAPU: "Es más fácil que pase un camello por el ojo de una aguja que entre un pobre a la UC" (Pontificia Universidad Católica). Hasta que el electo Presidente Allende llama al presidente de la FECH para que lo autorice a hablarle al país desde el balcón de la sede de los estudiantes... Patricio fue parte vital de estos tiempos posibles de sueños y cambios. Y, a pesar de todos los debates contingentes, tuvo el tiempo de soñar con su propio futuro al aprobar sus materias con regularidad.

Tiempos en que los estudiantes y profesores universitarios discutían cómo aportar a las trasformaciones deseadas, multiplicándose también por otras regiones. Entre los años 1967 a 1973 se gesta en el Instituto de Antropología de la Universidad de Concepción un cambio notable, en el cual el "grupo de los argentinos" y los locales fueron claves para innovar los marcos teóricos, incorporando al currículum, con Edgardo Garbulsky a la cabeza, junto a Julio Montané y Luis Lumbreras, el materialismo histórico. Por el norte acogíamos este desafío con materias que envolvían a la sociedad andina desde diversas miradas a través de la preparación, durante el año 1973, del Primer Congreso del Hombre Andino que obviamente, pareciera, será el último... Aquí Patricio tuvo un rol importante en la comisión organizadora.

\section{La Participación Institucional y sus Excavaciones}

Estoy seguro que sus primeros trabajos de campo los realizó en la costa central de Cachagua y Concón bajo la tuición de Bernardo Berdichewsky, donde bajo una cubierta estéril se identificó un conchal inesperado. Posteriormente, dada la conexión entre Junius Bird y Grete Mostny, puesto que ya estaba incorporado al Museo Nacional de Historia Natural, es enviado a la Patagonia para asistir a las excavaciones de la cueva Fell. Por otra parte, desde el año 1960 Julio Montané estaba a cargo de la sección de Prehistoria del MNHN, siendo acompañado por Patricio en sus excavaciones en el sitio paleoindio de Tagua Tagua (1967). En Julio encontró su guía adecuado, no solo para compartir lecturas relacionadas con materialismo histórico, sino porque en su entorno había un grupo de colegas notables: Virgilo Schiappacasse, Hans Niemeyer y Felipe Bate. No sabemos cómo se enrola en las excavaciones en la Isla de Pascua, pero William Mulloy tenía contactos con el Centro de Estudios Antropológicos de la Universidad de Chile, donde uno de los Munizagas lo habría recomendado.

Posteriormente, a raíz del Convenio entre la Universidad de Chile y la Universidad de California, Patricio es enviado, ya con todas sus materias aprobadas, desde el Centro de Estudios Antropológicos a nuestro Programa de Arqueología y Museo de la Universidad de Chile, sede Antofagasta. Junto con Delbert True condujimos el proyecto Caserones en la quebrada de Tarapacá entre los años 1966-1967. Fue un tiempo en que las publicaciones y aquellas aun por editarse, daban cuenta de la importancia de los temas abordados y de la calidad de los sitios. En esta relación puedo mencionar un hecho local excepcional, cuando nos dimos cuenta (ambos agnósticos...) que la iglesia colonial quemada estaba colmatada de escombros y sedimentos, y saqueada por coleccionistas foráneos. Excavamos allí con todo el equipo durante un par de semanas y la despejamos, recogiendo sus piezas de valor para entregarlas a los pocos vecinos que en ese entonces vivían en San Lorenzo. Finalizadas estas tareas le pusimos dos puertas sólidas y así cerramos la posibilidad de más intervenciones. Patricio decía que se había ganado el cielo con su propio oficio... 
Recorrimos este espacio tarapaqueño desde Pampa Iluga a Mocha para optar por el tramo inferior en torno a Caserones. Allí Patricio participó excavaciones con Delbert True en varios sitios arcaicos y con nosotros algunos recintos del poblado de Caserones. También se encargó de excavar las aldeas del Intermedio Tardío del sector de Huarasiña y el sitio monumental de Tarapacá Viejo con componentes del Intermedio Tardío, inca imperial y regional, además del trazado hispánico sobreimpuesto.

Compartimos aquí un largo tiempo, alojando en San Lorenzo de Tarapacá, de modo que para el Santo Patrono estos "agnósticos" fueron designados para ascender a la torre y hacerse cargo de las campanadas. Estábamos convencidos de que debían ser escuchadas desde muy lejos y desde lo más alto posible, a lo largo de toda la quebrada... Bajamos con las manos partidas como señal que habíamos convocado a las fuerzas de todos los panteones posibles para superar la sequía, como si de tanta convocatoria pudiera repoblarse la quebrada.

No podía ser de otro modo y en el año 1972 lo invitamos a formar parte estable de nuestro Programa de Arqueología y Museo en Antofagasta. Allí participó con el colectivo de nuestra Universidad de Chile en las excavaciones de Tiliviche y más particularmente en el asentamiento arcaico Aragón que quedó bajo su conducción junto a Vjera Zlatar. También fue muy activo en las excavaciones finales del cementerio Pica 8. En la costa al sur de Iquique se incorporó a las excavaciones estratigráficas de Cáñamo y continuamos hacia la desembocadura del río Loa (Caleta Huelén) en el año 1970, cuando prospectamos y muestreamos diversos sitios hasta concentrarnos en el asentamiento clave: Caleta Huelén-42. Allí desembarcamos en una goleta pesquera (no había caminos), y por su costumbre de andar a "cuero pelado" fue atacado de día por jerjeles y tábanos kamikazes y en la noche por nubes de zancudos que lo dejaron irreconocible. A pesar de todo tenía el coraje de cruzar el río, metiéndose en una avenida torrencial que lo cubría de banda a banda. Recuerdo que en esa misma noche nos quejamos de no contar allí con pescadores y mariscadores de orilla para entender las labores desde la etnografía, de la cual no sabíamos casi nada.

En el año 1973 nos abocamos con Patricio como miembros de la Comisión Organizadora al Primer Congreso del Hombre Andino. En ese año cumplíamos diez años de vida institucional. Patricio era parte del staff junto a las colegas Vjera Zlatar (antofagastina, magíster de la Universidad de Zagreb), Carolina Staal (Universidad de Holanda) y el Prof. de artes plásticas Branko Marinov, a su vez Conservador del Museo en Antofagasta. Lo complementaba el Prof. Alfredo Loayza del Museo de Iquique y el Prof. Carlos Fica a cargo del Museo de Calama. El equipo se incrementó con la contratación de Eduardo Muñoz, encargado del patrimonio cultural monumental, de los laboratoristas de restauración y conservación: Jaime Salazar y Françoise Mairet del Centro Nacional de Investigaciones Científicas de Francia, además del encargado de arte rupestre Prof. Patricio Moreno.

El golpe cívico militar repercutió fuertemente en la sede Antofagasta de la Universidad de Chile, por cuanto todas las autoridades fuimos apresadas y sometidas a sumario por la fiscal de la Casa Central, profesora de la Escuela de Derecho de nuestra Universidad, María Angélica Figueroa Quinteros. Un total de 42 académicos sin defensa jurídica fuimos exonerados con prohibición de pisar espacios universitarios del Estado. Patricio, aunque fue denunciado ante la Rectoría como marxista confeso logró sobrevivir con dignidad el momento de la razzia.

La misión de los rectores delegados era hacer desaparecer a las "peligrosas" sedes de la Universidad Técnica del Estado y de la Universidad de Chile. Así se creó en el año 1981 una nueva Universidad, la de Antofagasta, que debía integrar a ambas. Esto explica por que Patricio desde el año 1983 es parte del grupo de académicos que conforman lo que inicialmente se llamó Instituto de Investigaciones Arqueológicas y Restauración Monumental de dicha Universidad.

Patricio tomó al inicio la dirección del Instituto en la Universidad de Antofagasta y en buena hora logró retomar sus objetivos originales con importantes proyectos que pusieron énfasis en la conservación del patrimonio con la activa participación de Eduardo Muñoz, Branko Marinov y Vjera Zlatar. Paralelamente promovió el salvamento de los geoglifos de la segunda región y de las oficinas salitreras, incluyéndose en el año 1981 su participación en la expedición de las rutas prehispánicas entre Peine y Copiapó con Hans Niemeyer, Eduardo Muñoz y otros. En este tiempo se destaca muy particularmente su larga y compleja labor en torno a la conservación del pukara de Quitor (1988-1990).

Por el año 1993 y con fondos escuálidos se concentró con su equipo en los sitios formativos de Socaire, tratando de entender el rol y tiempo de ese complejo orden hidráulico, oportunidad en que esclareció la temporalidad de las terrazas y una expansión agraria reconocida como una hacienda de los incas. Junto a ello acogió el profundo cariño de la comunidad local, rasgo que lo conocía muy bien en su paso por la quebrada de Tarapacá. Una vez jubilado continuó preocupado por la arqueología costera al integrarse como investigador adjunto al Museo Augusto Capdeville Rojas de Taltal con el apoyo de su director Rodolfo Contreras. 


\section{Las Publicaciones}

Bajo el principio de la creatividad científica, o eso que se llama hoy "productividad académica" competitiva en los tiempos neoliberales, Patricio optó por su propio camino, buscando un equilibro entre la propuesta de datos duros con importantes aportes a la extensión o educación de la sociedad, tan válidos como aquellos que enfatizan la publicación con su mérito netamente científico. Ambas opciones son legítimas y complejas, y cada científico(a) asume su independencia frente al cómo expone sus investigaciones y apoya en más o en menos su difusión. Patricio optó por crear conocimientos de la misma manera como lograr su plena difusión. A continuación, se comentarán sus publicaciones en orden de secuencia.

Su primer aporte como coautor con Junius Bird ocurrió en el XXXIX Congreso Internacional de Americanistas de Lima (1970). En la convocatoria del Primer Congreso del Hombre Andino firma sus objetivos tocando un tema de su máximo interés: el materialismo histórico. Lo acompañaron Julio Montané y sus colegas de la Universidad de Chile de Antofagasta (1973). En ese mismo Congreso presentó su ponencia en el simposio 4: El rol de la sociedad andina en el tránsito al socialismo, titulada "En torno a problemas de la revolución cultural andina en el norte de Chile". Junto con Branko Marinov valoró posteriormente la relación entre la sociedad y el mar, vinculada con una exposición museográfica en el museo de Antofagasta (1975).

Publicamos junto con Patricio y Vjera los primeros resultados de las excavaciones en Caleta Huelén-42, seguido de un estudio sobre los movimientos transhumánticos entre la costa de Pisagua y Pampa de Tamarugal para evaluar posteriormente las relaciones prehistóricas trasandinas entre el noroeste argentino y norte de Chile (1976). En el mismo año Patricio con su colega Vjera publica los resultados de los análisis radiométricos del asentamiento arcaico de Aragón. A continuación, ambos valoraron los contextos (1976) y la coexistencia de las evidencias recolectoras y cazadoras (1978), mientras que plantearon las comparaciones entre Tiliviche y Aragón (1978).

Teniendo aun su cargo en la Universidad de Chile de Antofagasta, toca por primera vez la relación entre arqueología y restauración monumental, estimulado por los importantes proyectos conducidos por su colega Eduardo Muñoz, experto y miembro de ICOMOS (1976). Preocupación que reitera al interior de las ciencias sociales (1986). Un importante artículo sobre la economía prehispánica de Socaire lo acerca a este aislado oasis atacameño (1991). Otra publicación derivada del proyecto en Socaire dio cuenta del sistema de regadío inca y el potencial agrario del lugar y de la secuencia de las ocupaciones (1993). También abordó la religiosidad andina en los estudios realizados en Socaire (1994). Igualmente fue importante su prólogo de la reedición del "Glosario de la Lengua Atacameña" de E. Vaisse, F. Hoyos y A. Echeverría y Reyes en respuesta a las aspiraciones de la comunidad atacameña (2006). En un estudio posterior valora el rol del Museo de Augusto Capdeville (2008), al mismo tiempo que requería ante el Consejo de Monumentos Nacionales la protección de edificios patrimoniales como el caso del edificio de la Aduana de Antofagasta.

Su acercamiento al arte, que mantiene en su ADN, lo aplica en sus observaciones sobre el arte y artesanías prehispánicas (1996), tema que completa en otra publicación (1998), incluyendo en otro artículo sus orientaciones sobre el arte rupestre (1998). Su primer acercamiento al tema del género desde la vida prehispánica (1997) lo lleva a una mirada más profunda en un artículo premonitorio sobre una visión social del género a través de los 12.000 años de historia (1999), seguido ese mismo año de su percepción social de la historia para abordar en un estudio más detallado la relación entre arqueología y cambio social desde una perspectiva del género ajustado al materialismo histórico, utilizando datos del norte de Chile (2000). Efectivamente, Patricio se adelantó a los tiempos, cuando en el año 1999 expuso la importancia de las reivindicaciones del género, ahora vigentes, al indicar que:

Consideramos que el materialismo histórico está vigente en el campo de las ciencias sociales para interpretar los procesos de cambio. En esta oportunidad, al tratar los cambios sociales que se produjeron en la prehistoria del Norte de Chile, consideramos para su interpretación conceptos de la visión de género. Pensamos, que, si bien ha existido una preocupación del papel de la mujer en la historia, especialmente en los momentos de crisis, ha sido una visión patriarcal o masculina que ha impedido un mejor conocimiento de la problemática social y de los roles en la producción.

Presenta su análisis sobre el poder de las comunidades indígenas (2000), precedido por la ponencia muy ajustada a su interés por el arte: "Estudio de arte y artesanía prehispánicas en el Norte de Chile" (1998). Al tanto que el tema sobre identidad y prehistoria lo centra en la primera y segunda región (2001). Su gradual acercamiento con más preferencia a los temas marítimos valora la naturaleza de vida en el mar "para vivirlo" 
(2003) y, siguiendo con el género (2003), retorna a sus reflexiones, esta vez sobre el vivir y morir en los Andes (2005). Sin embargo, vuelve a la revolución agropecuaria, un aporte que lo venía manejando desde el Congreso del Hombre Andino y que calzaba muy bien con su enfoque "Childeano" (2004). Los análisis históricos se hicieron presentes, cuando publicó la naturaleza de las dos tradiciones, incas y españolas, durante el siglo XVI.

Patricio últimamente gustaba aplicar el término "sueño" como un sinónimo de ideario, aquel que anhelamos como aquellos cambios que deben ayudar al progreso social de la humanidad. Fue así que escribió sobre el sueño de la ilustración del siglo XVI en el norte de Chile (2016). Pero su último libro fue más explícito, lejos su proyecto más personal con esa originalidad propia de su sensibilidad que venía del arte, absorbido desde su infancia en una casa donde el arte y materialismo histórico eran sustancia de la cotidianidad. Su título: "Vivir después de Soñar" (2016) podría haberlo aplicado a su propia autobiografía...Volvió al tema a raíz de su estudio del sitio Punta Negra, ubicado en la costa cerca de Taltal, donde se identificaron bloques y rocas móviles con enigmáticas incisiones que lo llevaron a caracterizar este sitio como un "espacio y tiempo para soñar" (2017). Estaba orgulloso de su obra, porque unía arqueología y arte, y lo vinculaba con sus tradiciones familiares. Al igual que el poeta Pablo de Rokha entregaba su publicación personalmente a sus amigos más cercanos.

Patricio asumió que la comunidad arcaica de Punta Negra desconoció realmente el origen de los fenómenos de la naturaleza, pero él, conociéndolos, creyó en los sueños hasta el final, donde su profundo humanismo y espiritualidad pudieron convivir con su visión materialista de la historia. No en vano elige esta cita de Ambrogio Donini (1961:26): "Los sueños, el estado de sueño y la muerte sirven para explicar el origen de los fantasmas religiosos y de las primeras ideas del alma".

Este libro nace con el descubrimiento del sitio en Punta Negra (2010), reconocido ahora como un centro ceremonial arcaico (6000-4000 a.p.) que lo llevó, por su adicción a la lectura, a citar a numerosos autores desde el siglo XVIII hasta ahora. Para comprender estas líneas incisas invoca el arte y la estética hasta vincular ideología entre pasado y presente. Al respecto, realizamos comparaciones con las líneas similares arcaicas formativas del transecto Tulán, y nos entusiasmamos con posibles interacciones entre la circunpuna y la costa. Yo lo escuché detenidamente, porque él avanzó muchísimo en colocar su particular mirada sensible entre pasado y presente, donde vinculaba las fuerzas productivas con la creatividad y la espiritualidad propias de los rituales visuales del pasado. Señalaba que los humanos en todos los tiempos lo habían logrado a través de abstracciones homologas, aun en el arte contemporáneo, hasta involucrar a las pinturas de su propio hermano Guillermo...

Su libro termina como su vida, aceptando que en toda la humanidad ha existido esa religiosidad como aquellas rocas sagradas, en tanto que los habitantes de Punta Negra, como el mismo dice: "hacen suya la ritualidad ceremonial, participando, creyendo. Viviendo después de soñar"... Es que esta obra se ajusta exactamente a sus idearios. En el prólogo se encuentra su leitmotiv crucial: "Lo bello en el tiempo y en el espacio antes de vivir y soñar", de donde se desprende que se habla a sí mismo, recordándose cuál ha sido su propia norma de vida: "Después de soñar $\mathrm{y}$ tener por lo tanto nuevos pensamientos e ideas, se despierta, se vive y se sueña nuevamente para seguir viviendo" (2016:18).

\section{Epílogo}

Siendo un gran lector de las propuestas de la arqueología social latinoamericana, éstas fueron uno de los temas principales del Congreso Andino que organizamos en Arica, Iquique y Antofagasta con un discurso inaugural brillante de parte de Alejandro Lipschutz. Hasta que el golpe cívico militar nos marginó de este diálogo esencial recientemente compilado por H. Tantaleán y M. Aguilar. Se había frustrado este fuerte movimiento con el exilio de sus principales protagonistas. Sin embargo, Patricio, leal a su dialéctica materialista histórica, no dejó de reflexionar sobre cómo preservar el marxismo en sus pensamientos y escritos. Y ahora se despide, cuando más se le necesita por su lealtad a un ideario debatido que jamás abandonó. Esto explica una de las pocas veces que lo vi alterado, estado poco común en él de acuerdo a su permanente cordialidad, cuando comentó que le molestaba leer a investigadores acrobáticos que saltan de una escuela de pensamiento a otra al compás del redoble de los modelos de moda. A continuación conversamos sobre qué se debe publicar de acuerdo a las exigencias para levantar las acreditaciones de nuestras universidades versus lo que aspiramos desde nuestras propias convicciones. Me indicó que desde su formación social era muy importante publicar los textos de extensión o educación hacia las mayorías, que directa o indirectamente son segmentos de nuestros largos procesos históricos de investigación, generalmente desconocidos. Que el ideal era repartir los tiempos entre los análisis y su difusión, aspecto que recién hoy se tiende a valorar más que antes. Yo estaba de acuerdo y le decía que de esta manera se 
debería juzgar a los arqueólogos que nos precedieron, de acuerdo a sus tiempos y sus marcos teóricos, en ese contexto temporal, con independencia de si lograron o no comunicar sus resultados a todo público. En este sentido, advertíamos que ahora aun los análisis empíricos son importantes en la medida que son difundidos al interior de las comunidades científicas, necesitándose de más esfuerzos, como los de Patricio, para lograr una debida divulgación. Sin embargo, recuerdo haberle dicho algo así que lo aceptamos plenamente, vislumbrándose en su rostro su particular sonrisa explosiva: "Tanto en ciencia como en amor los excesos analíticos sin la comprensión de la sociedad que nos rodea podrían conducirnos peligrosamente a la impotencia"...

Después del golpe militar, al ser exonerado de la Universidad de Chile en Antofagasta en el año 1974, dejé de ver a Patricio y sé que debió mantener con delicadeza las labores programáticas del Instituto, sobre todo cuando se integra a la Universidad de Antofagasta. Sus actividades empiezan a coexistir con otras disciplinas sin abandonar su pasión por la disciplina arqueológica desde su particular percepción materialista.

Patricio fue un genuino soñador profesional y suspendió su estado de ensoñación desde la realidad social el 10 de noviembre de 2017. Lo despedí un día antes junto a Rodrigo, hijo de Inger Kock (Carolina venía en viaje), y su compañera Adriana Sáez. Desde su plena lucidez me habló de nuestro bello tiempo compartido y le retribuí en esa misma dirección, recordándole, además, que siempre fue nuestro colega más "regalón", oportunidad en que se acercó Adriana... para ratificarlo, tomándole también sus manos. Luego le dije que nos encontraríamos por ahí en algún sitio con sus grabados en surcos para festejarnos como se debe... Le vi su particular sonrisa en los momentos en que cerraba los ojos. Guardé silencio. Pensé que esperaba una despedida. Me miró de nuevo y solo atiné a recordar nuestros viejos tiempos: Levanté mi puño izquierdo en alto y desde abajo lo replicó de inmediato. Se fue con sus sueños intactos...

\section{Referencia Citada}

Huidobro, V. 1948. El paso del retorno. En De Últimos Poemas. Póstumo.

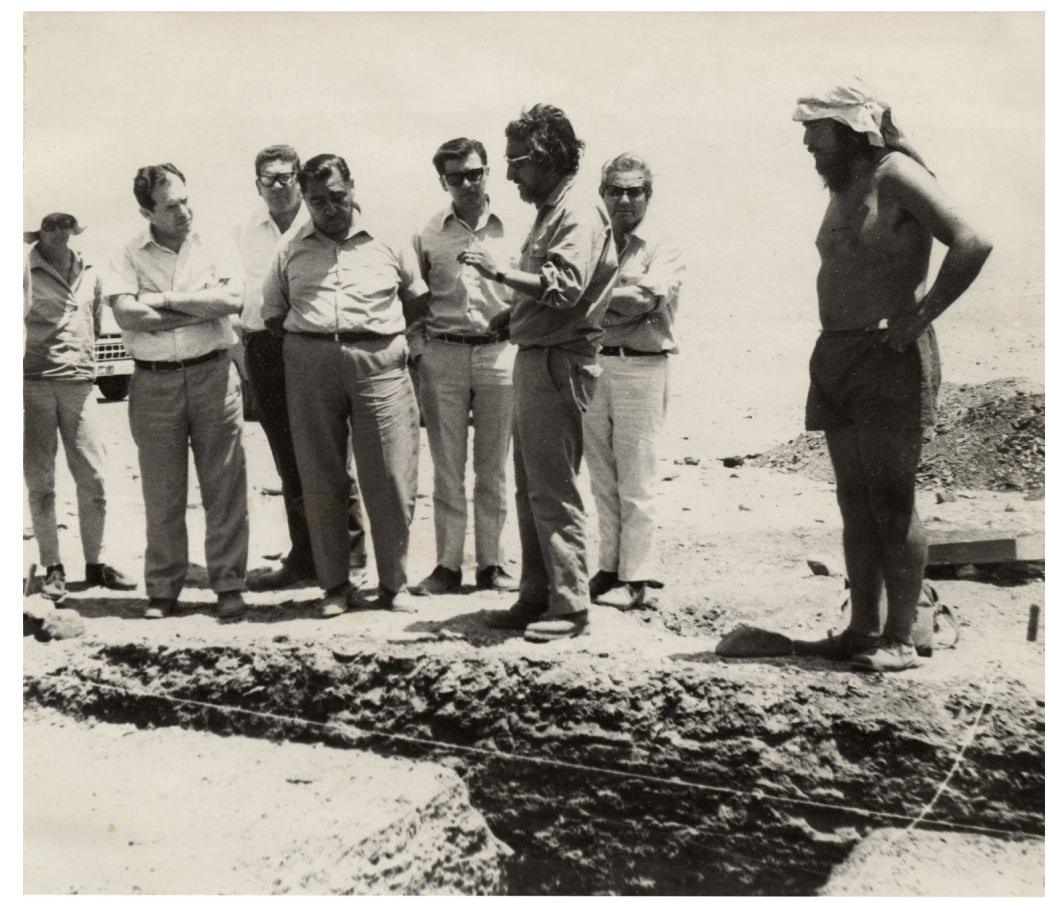

Excavaciones en Caleta Huelén-42: A la derecha Patricio Núñez, en el centro Lautaro Núñez explica los procedimientos al intendente de Tarapacá Alejandro Soria junto a autoridades de Iquique, a la izquierda Vjera Zlatar, 1971 (fotografía cortesía de Lautaro Núñez). 


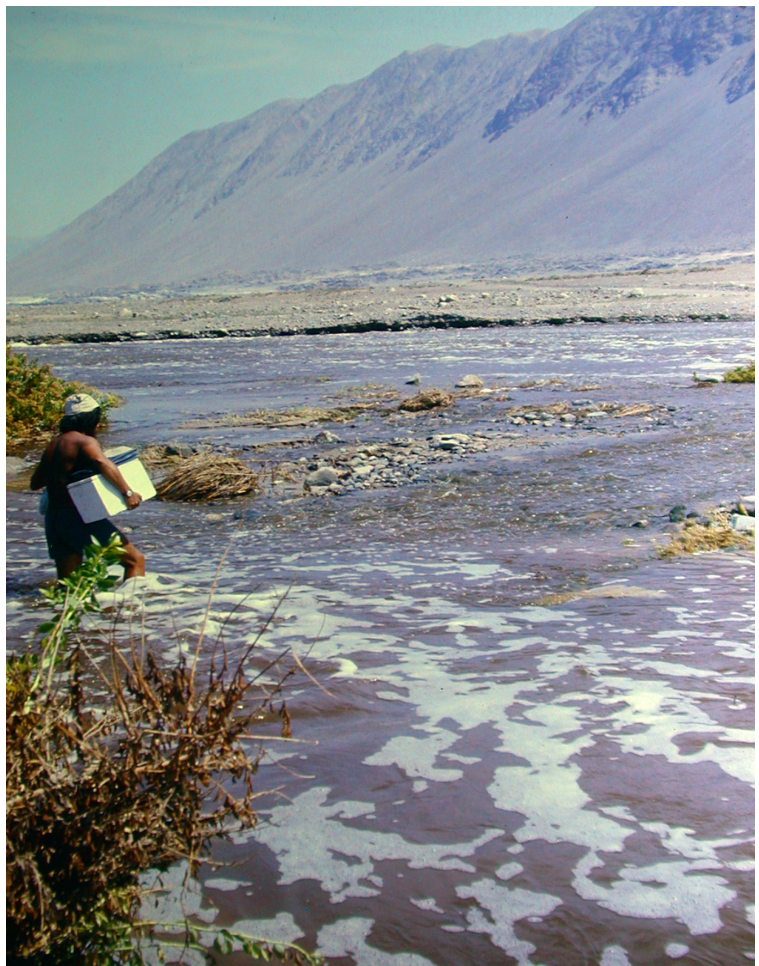

Patricio Núñez cruzando la desembocadura del río Loa afectada por una avenida torrencial que cubrió ambos bordes durante las excavaciones en Caleta Huelén-42, 1971 (fotografía cortesía de Lautaro Núñez). 